\title{
New Evidence for the Mixing Length Concept in a Narrow Bubble Column Operated in the Transition Regime
}

\author{
Stoyan Nedeltchev ${ }^{1}$, Thomas Donath ${ }^{2}$, Swapna RabHa ${ }^{2}$, \\ Uwe HAMPEL ${ }^{2,3}$ and Markus SCHUBERT ${ }^{2}$ \\ ${ }^{1}$ Institute of Technical Chemistry, Technical University of Braunschweig, \\ Hans-Sommer-Strasse 10, 38106 Braunschweig, Germany \\ ${ }^{2}$ Institute of Fluid Dynamics, Helmholtz-Zentrum Dresden-Rossendorf, \\ Bautzner Landstraße 400, 01328 Dresden, Germany \\ ${ }^{3}$ AREVA Endowed Chair of Imaging Techniques in Energy and Process Engineering, \\ Dresden University of Technology, 01062 Dresden, Germany
}

\begin{abstract}
Keywords: Narrow Bubble Column, Wire-Mesh Sensor, Gas Holdup Fluctuations, Mixing Length, Transition Flow Regime, Maximum Number of Visits, Kolmogorov Entropy

Different scales of liquid mixing exist in bubble columns and it is very important to determine the prevailing mixing scale in each flow regime. Two independent parameters were found to exhibit a monotonous decline in the transition flow regime, which could be attributed to the decrease of the mixing length values $L$. In this work, a new parameter called "maximum number of visits in a region" $\left(N_{\mathrm{v}}{ }^{\max }\right)$ and the Kolmogorov entropy $(K E)$ were extracted from the gas holdup time-series $(60,000$ points). The latter were recorded at a high sampling frequency $(2,000 \mathrm{~Hz})$ by a wire-mesh sensor. The measurements were performed in a narrow bubble column $(0.15 \mathrm{~m}$ in i.d., clear liquid height $=2 \mathrm{~m})$ equipped with a perforated plate distributor ( 14 holes, $\varnothing 4 \times 10^{-3} \mathrm{~m}$ ). Both parameters were capable of identifying concordantly the two main transition velocities at $U_{\text {trans }}=0.022$ and $0.112 \mathrm{~m} / \mathrm{s}$, which delineate the boundaries of gas maldistribution, transition and churn-turbulent regimes, respectively.
\end{abstract}

\section{Introduction}

In bubble columns, gas is dispersed into a volume of liquid by means of a gas distributor to achieve good interphase mass transfer. The liquid flow induced by the rise of bubbles is very complicated and affects strongly the column performance. Bubble column hydrodynamics are characterized by different flow patterns depending mainly on gas flow rate, physico-chemical properties of the gas-liquid system, gas distributor design, column diameter, etc. (Ajbar et al., 2009).

The understanding of the mechanism and the knowledge about the magnitude of the liquid-phase mixing in bubble columns is very important for a successful design of these gas-liquid reactors. It is well-known that the bubble sizes and their rise velocities are the most important hydrodynamic parameters which govern gas holdup, liquid mixing, mass and heat transfer rates. The turbulence intensity is also of essential importance since it has a pronounced influence on the local transfer rate. In the bottom region (close to the gas distributor) the turbulence intensity is higher than in the rest of the column (Nedeltchev, 2009). At high super-

Received on February 27, 2014; accepted on March 3, 2014

DOI: $10.1252 /$ jcej.13we362

Correspondence concerning this article should be addressed to $\mathrm{S}$. Nedeltchev (E-mail address: snn13@gmx.net).

Presented at the DECHEMA Annual Meeting of the Groups of Multiphase Flows and Heat and Mass Transfer, 24-25 March, 2014, Fulda (Germany). ficial gas velocities $U_{\mathrm{G}}$ the liquid phase is highly turbulent throughout the bubble bed.

The degree of liquid mixing depends on the prevailing flow regime. In a bubble column, each flow regime transition is as a function of the physical properties of gas and liquid phases and of the system specifications (gas distributor design, column dimensions, etc.). Therefore, it is essential to know the range of parameters over which a particular regime prevails and the conditions under which the transition occurs.

Bubble columns can be operated in homogeneous, transition or heterogeneous flow regimes. The boundaries of these regimes are distinguished by two transition velocities $U_{\text {trans. }}$. The bubble behavior and the degree of liquid mixing are different in each flow regime. The bubble column performance is affected by both the flow regime and the quality of the gas distribution.

The homogeneous (dispersed bubble) flow regime is encountered at low $U_{\mathrm{G}}$ values when the gas from the sparger is uniformly distributed (Ajbar et al., 2009). It is characterized by gentle agitation of the gas-liquid dispersion by relatively small spherical bubbles of small size. The bubble size distribution is very narrow and depends only on the gas sparger. The bubble streams are observed to rise rectilinearly. Bubble coalescence is insignificant. A relatively uniform cross-sectional gas holdup distribution and a rather flat liquid velocity profile are observed. In this flow regime, both the gas distributor and the properties of the gas-liquid system play a dominant role in the evolving bubble-size distribution and 
in the gas holdup profile (Ajbar et al., 2009). When a perforated plate distributor with large openings is used, then the quality of the gas distribution is poor and a gas maldistribution regime is established (usually at low $U_{\mathrm{G}}$ values). Even well-designed bubble columns encounter gas distribution problems as the gas distributor becomes plugged. The gas maldistribution across the cross-sectional area of the bubble column causes density differences which induce a liquid circulation pattern (Groen et al., 1996). The determination of the upper boundary of the gas maldistribution regime is very important.

The transition flow regime is characterized by large flow macro-structures (large eddies and global liquid flow macro-structure) and widened bubble size distribution due to the onset of bubble coalescence. The latter occurs mainly near the gas sparger. This regime corresponds to the development of local liquid circulation patterns in the column. It is well-established that the occurrence and the persistence of the transition regime depend largely on the uniformity and the quality of the aeration.

As $U_{\mathrm{G}}$ increases, larger bubbles start to form as a result of the coalescence of small bubbles whose wakes cause gross circulation patterns in the bubble bed resulting in a heterogeneous (churn-turbulent) flow regime. The transition from homogeneous to heterogeneous flow regime is a gradual process. The heterogeneous regime is characterized by the wide distribution of bubble sizes and by the existence of a distinctive radial gas holdup profile, which causes liquid circulation. The hydrodynamics in this flow regime are more influenced by the bubble-induced turbulence (Ajbar et al., 2009). In this hydrodynamic regime, coalescence and breakup occur. Bubbles agglomerate in the vicinity of the gas distributor to form larger bubbles. The heterogeneous flow regime is characterized by vigorous mixing and coherent structures (bubble swarms, circulation cells, etc.). It is argued that in this flow regime the gas distributor has a minor influence.

Our work is focused on three problems:

- the detection of the gas maldistribution regime

- the identification of the boundaries of the main flow regimes

- the determination of the scale of liquid mixing in the transition flow regime

\section{Mixing Length Concept}

According to Geary and Rice (1992) the appropriate length scale of turbulence for small columns should be based on bubble diameter $d_{\mathrm{b}}$, while for larger columns the proper mixing length is proportional to the column diameter $D_{c}$. Lübbert and Larson (1990) argued that the dominant mixing mechanism arises from bubble wakes. Deckwer et al. (1974) and Baird and Rice (1975) developed correlations in which the liquid-phase axial dispersion coefficient $E_{\mathrm{L}}$ is correlated to $D_{c}$. These correlations are mainly valid in the churn-turbulent flow regime. Baird and Rice (1975) applied the Kolmogorov's theory of isotropic turbulence and used $D_{c}$ as a characteristic eddy dimension (approximate diameter of large vortices). In the homogeneous flow regime, however, Rice and Littlefield (1987) correlated $E_{\mathrm{L}}$ to $d_{\mathrm{b}}$. The authors argue that in this regime the bubble diameter should be used as a characteristic eddy dimension.

The concept of mixing length $L$ is a part of the mixing length theory explained by Schlichting (1968). The characteristic mixing length provides a useful basis for the evaluation of the hydrodynamics of the liquid phase. This concept is expected to facilitate the modeling of liquid-phase mixing in bubble columns. The liquid velocity at the column axis, $E_{\mathrm{L}}$ and the gas holdup can be theoretically derived based on this concept (Kawase and Tokunaga, 1991).

The choice of a correct mixing length scale is an important issue. Rice and Geary (1990) and Geary and Rice (1991) argue that the mixing length scale should be proportional to the bubble diameter $d_{\mathrm{b}}$. In this case the local mixing is dominating. Support for a bubble-based mixing-scale can be found in Lübbert and Larson (1990). Another physical interpretation of the mixing length is that it is the distance over which a turbulent eddy retains its identity.

According to Hinze (1975) "the mixing length should be proportional to the width of the turbulent mixing zone". Geary and Rice (1992) argue that small columns and columns with liquids of higher viscosity tend to be dominated by bubble-generated turbulence.

Kawase and Moo-Young $(1986,1987)$ have applied the mixing length theory to discuss the hydrodynamics in bubble columns. The authors developed theoretical models for the liquid velocity at the column axis as well as for $E_{\mathrm{L}}$ and the gas holdup. Later, Kawase and Tokunaga (1991) improved their concept of a characteristic mixing length. They examined the validity of the characteristic mixing length concept by considering also the measured radial liquid velocity profiles. Kawase and Tokunaga (1991) argued that although the mixing length shows a variation with radial position due to radial profile of the liquid velocity, a mean value across the column cross-section is still reliable and also more usable. They found that the value of the characteristic mixing length is not constant and decreases as the superficial gas velocity $U_{\mathrm{G}}$ increases:

$$
L=0.045 D_{\mathrm{c}} U_{\mathrm{G}}{ }^{-0.38}
$$

The inverse dependency of mixing length on $U_{\mathrm{G}}$ was attributed to the existence of bubbles and their complicated motion. It is noteworthy that Pandit and Joshi (1986) found that the scale of turbulence is proportional to $0.08 D_{c}$, so the mixing length in Eq. (1) is proportional to both scale of turbulence and $U_{\mathrm{G}}{ }^{-0.38}$.

The identification of the boundaries of the main flow regimes is a very important first step in order to determine the scale of liquid mixing in every flow regime. A new parameter for flow regime identification should be introduced and compared with the other available parameters (for instance, the Kolmogorov entropy). The profiles of these parameters in every flow regime should be examined and correlated to the bubble diameter or mixing length, i.e. the scales of liquid 

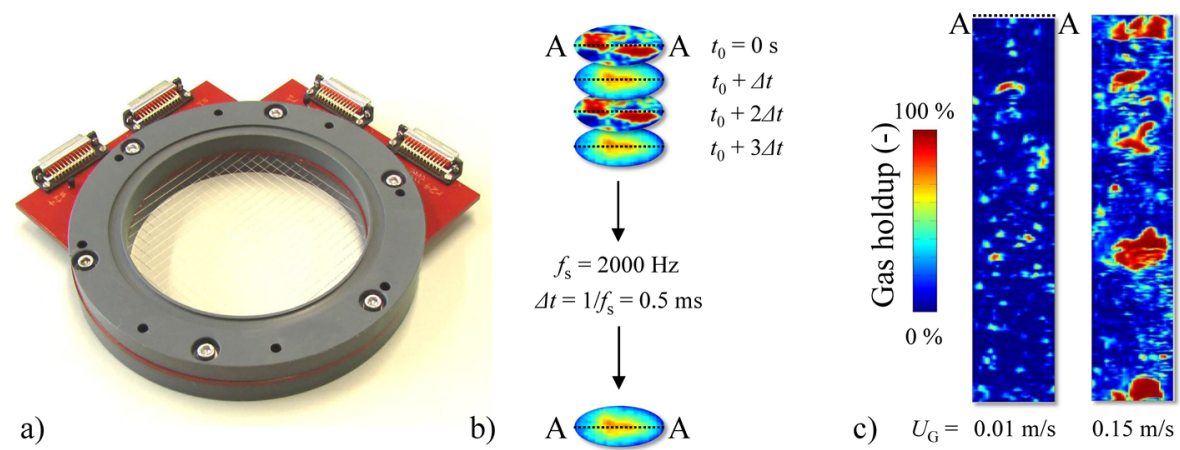

c) $U_{\mathrm{G}}=0.01 \mathrm{~m} / \mathrm{s} \quad 0.15 \mathrm{~m} / \mathrm{s}$

Fig. 1 Detailed description of the wire-mesh sensor; a) Photograph of the applied wire-mesh sensor, b) Visualization of the gas holdup in the column cross-section, and c) Virtual side $(A \cdots A=$ diameter) projection of exemplary measurements (vertical coordinate in time $=$ here $3 \mathrm{~s}$ )

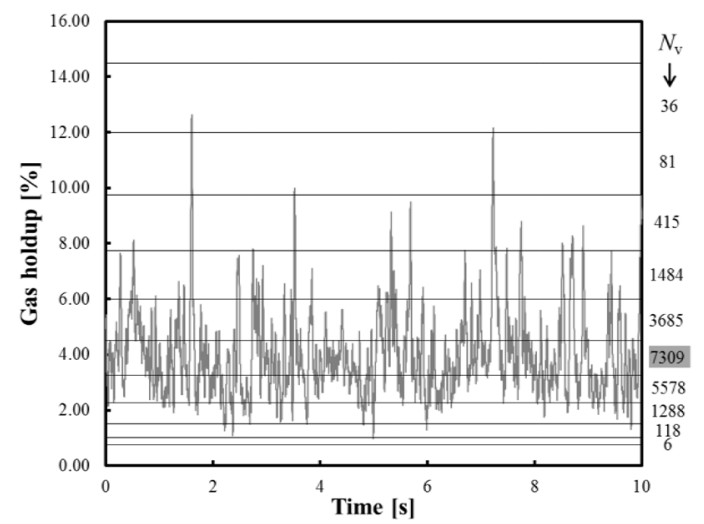

Fig. 2 Example of the cross-sectional average gas holdup time-series data $(20,000$ points $(10 \mathrm{~s}))$ obtained by the wire-mesh sensor $\left(f_{\mathrm{s}}=2,000 \mathrm{~Hz}\right)$ at $U_{\mathrm{G}}=0.012 \mathrm{~m} / \mathrm{s}$ in a narrow bubble column $(0.15 \mathrm{~m}$ in i.d.) operated with an air-water system

mixing in the different regimes. In order to obtain optimum results, the most suitable signal (for instance gas holdup time-series) should be selected.

\section{Experimental Setup}

The gas holdup time-series data were obtained in a narrow bubble column $(0.15 \mathrm{~m}$ in i.d., clear liquid height: $2.0 \mathrm{~m})$ equipped with a perforated plate distributor (14 holes with diameter $\varnothing 4 \times 10^{-3} \mathrm{~m}$ and open area of $1 \%$ ). The gas holdup was measured by a conductivity wire-mesh sensor installed at a height of $1.3 \mathrm{~m}$ above the distributor plate. It is worth noting that the raw gas holdup values were recorded in percentage [\%] and they were treated in this form by our methods of analysis.

The wire-mesh sensor (Figure 1(a)) consists of two electrode planes each with 24 stainless steel wires of $0.2 \times 10^{-3} \mathrm{~m}$ diameter and $6.125 \times 10^{-3} \mathrm{~m}$ distance between the wires. The distance between the planes is $4.0 \times 10^{-3} \mathrm{~m}$ and the wires from different planes run at right angles to each other. This arrangement gives 576 crossing points, thereof 452 inside the circular cross-section of the column. One plane of electrodes acts as a transmitter, the other as a receiver plane. The transmitter electrodes are activated by a multiplexing

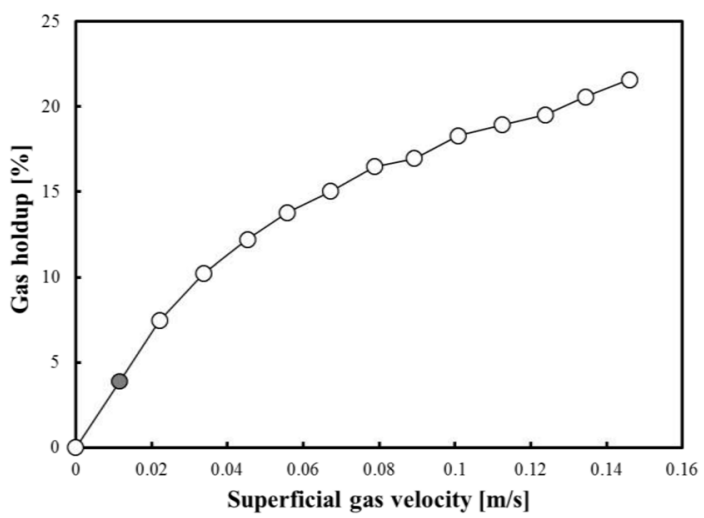

Fig. 3 Gas holdup profile as a function of superficial gas velocity in a narrow bubble column $(0.15 \mathrm{~m}$ in i.d.) operated with an airwater system

circuit in a successive order and signals derived from measured current at the receiver electrodes are stored. After one multiplexing cycle, a two-dimensionless matrix of values is available reflecting the conductivities between all crossing points of the electrodes of the two perpendicular planes. Details on the wire-mesh sensor circuit diagram can be found elsewhere (Prasser et al., 1998). The signals of the matrix can be converted into gas holdup data due to proper calibration measurements with liquid-flooded and empty column, respectively. Air was used as the gas phase and deionized water as the liquid phase.

The superficial gas velocity $U_{\mathrm{G}}$ was varied from 0.01 to $0.15 \mathrm{~m} / \mathrm{s}$. At each $U_{\mathrm{G}}$ value distribution of gas holdup in the cross-section was measured with a sampling frequency $f_{\mathrm{s}}$ of $2,000 \mathrm{~Hz}$ (see Figure 1(b)) over a measurement period of $30 \mathrm{~s}$. Such a high-frequent measurement allows visualization of the gas-liquid flow structures shown as virtual side projections in Figure 1(c).

Time-series of the gas holdup with 60,000 points for every $U_{\mathrm{G}}$ value was obtained by cross-sectional averaging of the matrix data. It is worth noting that the time-series are characterized with a low number of crossings of the mean value ( 817 crossings at $U_{\mathrm{G}}=0.012 \mathrm{~m} / \mathrm{s}$ down to 345 crossings at $U_{\mathrm{G}}=0.15 \mathrm{~m} / \mathrm{s}$ ). In other words, the average cycle time varies from 0.073 up to $0.174 \mathrm{~s}$. Example of the cross-sectional 
average gas holdup time-series data $(20,000$ points $(10 \mathrm{~s}))$ at $U_{\mathrm{G}}=0.012 \mathrm{~m} / \mathrm{s}$ is shown in Figure 2 .

The mean gas holdup values (averaged over the cross-section and over time) are presented in Figure 3. The averaged gas holdup value corresponding to the condition shown in Figure 2 is highlighted. It is clear that Figure 2 cannot be used for flow regime identification.

After selecting the most suitable signal (gas holdup timeseries) for flow regime identification and determination of the scale of liquid mixing, the methods for extracting of the hidden information in the time-series should be explained.

\section{Methods of Analysis}

\subsection{Maximum number of visits in a region $\left(N_{v}{ }^{\max }\right)$}

At first, the minimum and maximum values in the signal of each gas holdup time-series have been determined. Then, the signal's range has been divided into different regions with progressively increasing heights: $0.25,0.5,0.75,1.0$, $1.25,1.5$, etc. The raw gas holdup time-series are in percentage [\%]. The height of the smallest region (0.25) was used arbitrarily as the unit height (division step). The height of each subsequent region is proportional to 0.25 and the multiplication factor increases with unity, i.e. it is $1 \times 0.25$, $2 \times 0.25,3 \times 0.25,4 \times 0.25$, etc. A new parameter called "maximum number of visits in a region" $N_{\mathrm{v}}{ }^{\max }$ was defined and successfully used for the identification of the flow regime transitions in a narrow bubble column. $N_{\mathrm{v}}{ }^{\mathrm{max}}$ is equal to the number of counts in the most frequently visited region. The division into regions and the number of visits $N_{\mathrm{V}}$ in these regions (as described above) at $U_{\mathrm{G}}=0.012 \mathrm{~m} / \mathrm{s}$ are shown schematically in Figure 2. For simplicity and better illustration only part of the signal $(20,000$ points $(10 \mathrm{~s}))$ is shown. In this particular case, the fifth region is the most frequently visited one and therefore $N_{\mathrm{v}}^{\max }$ is equal to the number of counts (highlighted in grey) in this region. It is noteworthy that the number of visits in each region $N_{\mathrm{v}}$ is calculated by a computer program. The above-described concept is usually used as a basis for the application of the information entropy algorithm (Nedeltchev and Shaikh, 2013). However, in the case of gas holdup time-series measured by a wire-mesh sensor, $N_{\mathrm{v}}{ }^{\max }$ gives better results (for flow regime identification) than the information entropy.

\subsection{Kolmogorov entropy (KE)}

The bubble column can be regarded as a chaotic system, i.e. a system, which is governed by nonlinear interactions between the system variables. This nonlinear system is sensitive to small changes in the initial conditions and is, therefore, characterized by a limited predictability. The Kolmogorov entropy $(K E)$ characterizes the degree of unpredictability of the system. The $K E$ value reflects the rate of information loss of the system and thus, accounts for the accuracy of the initial conditions that is required to predict the evolution of the system over a given time interval. $K E>0$ is a sufficient condition for chaos and to some extent the chaotic system is only predictable over a restricted time in- terval. $K E$ is large for very irregular dynamic behavior, small in the case of more regular, periodic-like behavior, and zero for completely periodic systems. This parameter is sensitive to changes in the operating conditions and thus, it can be employed for flow regime identification (Letzel et al., 1997).

The $K E$ values were calculated on the basis of the algorithm developed by Schouten et al. (1994a). This algorithm detects the main transition velocities which correspond to flow instabilities. It extracts the dynamic information hidden within the time-series. The $K E$ is calculated from the average number of steps required for an arbitrary pair of vectors $X_{i}$ and $X_{j}$, which are initially within a specific maximum length $L_{0}$, to separate (exponentially) until the distance between the pair becomes larger than $L_{0}$. More information about the random generation of vector pairs can be found elsewhere (van den Bleek and Schouten, 1993; Schouten et al., 1994a, 1994b). Generally speaking, the maximum-likelihood of the $K E$ can be expressed as follows:

$$
K E=-f_{\mathrm{s}} \ln \left(1-\frac{1}{\bar{b}}\right) \text { with } \bar{b}=\frac{1}{M} \sum_{i=1}^{M} b_{i}
$$

where $f_{\mathrm{s}}$ is the sampling frequency $\left(f_{\mathrm{s}}=2,000 \mathrm{~Hz}\right.$ in this work). At first, an initial pair of independent (without repeating elements) vectors $X_{i}$ and $X_{j}$ within a maximum interpoint distance $L_{0}$ is selected. The variable $b$ equals the number of sequential pairs of points, in which the interpoint distance is for the first time bigger than the specified $L_{0}$. In the present analysis, the distance between two reconstructed state vectors $X_{i}$ and $X_{j}$ was estimated on the basis of the maximum norm definition (Schouten et al., 1994a, 1994b). A very high number of pairs of vectors have been generated. Based on them the number of steps $b$ have been calculated and ultimately the average number $M$ has been estimated.

Each state vector is reconstructed from the data points in the time-series. This is done by choosing a specific delay time $\tau$ in discrete units of equal sequential time steps between the elements of the state vector and the number of elements $m$ of the state vector (van den Bleek and Schouten, 1993). The number of elements $m$ of the state vector is called embedding dimension and it is equal to the number of coordinates in the reconstructed state space. In our research, the delay time $\tau$ is set equal to 1 and the embedding dimension $m$ is set equal to 50 as recommended by Letzel et al. (1997).

The distance between two initially close vectors should be compared with some prescribed distance (so-called "cutoff" distance or length $L_{0}$ ). In calculating the $K E$, the rate of growth of the distance between two initially close vectors is considered. The KE algorithm is focused specifically on distances that are smaller than a specific "cut-off" distance $L_{0}$ (called also maximum "scaling" distance). Letzel et al. (1997) suggested that the cut-off length $L_{0}$ should be set equal to 3 times the average absolute deviation (a robust statistical measure of the data width around the mean value). The choice of $L_{0}$ is actually a matter of empiricism because no clear-cut theoretically founded rule (for $L_{0}$ selection) is available (Schouten et al., 1994b).

Each $K E$ value was extracted from $N=60,000$ points, 
which was a time-consuming process. According to van den Bleek and Schouten (1993), for $N$ data points principally $N-m+1$ reconstructed state vectors can be obtained. So, following this principle in our work the number of $b$ values at every operating condition was higher than $N$. In our research the $b$ values were computed on the basis of a comparison of the distance between numerous randomly generated vectors (in different parts of the time-series) which consisted of elements located not farther than 10,000 points from each other in the raw time series.

More information about the application of the nonlinear chaos analysis can be found elsewhere (van den Bleek and Schouten, 1993; Schouten et al., 1994a, 1994b).

\section{Results and Discussion}

In this work, the maximum number of visits $N_{\mathrm{v}}{ }^{\max }$ in a single region is introduced as a new concept for the identification of flow regime transitions. Figure 4 shows that the $N_{\mathrm{v}}{ }^{\max }$ values as a function of the superficial gas velocity $U_{\mathrm{G}}$ can successfully identify the two main transition velocities $U_{\text {trans }}$ in a narrow bubble column $(0.15 \mathrm{~m}$ in i.d.) operated with an air-water system. At $U_{\mathrm{G}}=0.022 \mathrm{~m} / \mathrm{s}$ there is a distinct local minimum, which identifies the end of the gas maldistribution regime and the beginning of the transition flow regime. At every transition velocity some reorganization occurs and more structure is observed as suggested by Letzel et al. (1997). Every transition point should be regarded as the onset of the new flow regime. In other words, in every transition point an old structure is being destroyed and a new one is being formed. This leads to a transition from a less ordered state (old flow regime) to a more ordered state (new flow regime). Therefore, at every transition velocity the signal becomes more uniformly distributed into different regions and the maximum number of visits in a single region $N_{\mathrm{v}} \max$ drops.

This critical gas velocity is close to the one $(0.029 \mathrm{~m} / \mathrm{s})$ predicted by the correlation of Reilly et al. (1994). The second local minimum in the $N_{\mathrm{v}}^{\max }$ profile occurs at $U_{\mathrm{G}}=0.112 \mathrm{~m} / \mathrm{s}$ and it marks the onset of the churn-turbulent flow regime. The two critical gas velocities $U_{\text {trans }}$ identify the boundaries of a wide transition flow regime. Figure 4 shows that in every flow regime the $N_{\mathrm{v}} \max$ values monotonously decrease. Except for the two transition velocities, the $N_{\mathrm{v}}^{\max }$ values are decreasing steadily as a function of $U_{\mathrm{G}}$. The existence of two $U_{\text {trans }}$ values will be confirmed later by means of the $K E$ profile.

The existence of gas maldistribution is clearly visualized by photos. Figure $\mathbf{5}$ shows a typical gas maldistribution at $U_{\mathrm{G}}=0.01 \mathrm{~m} / \mathrm{s}$. It is clearly seen that the gas does not pass (no bubble formation) through some of the perforations at the gas distributor plate. Operating bubble columns in a gas maldistribution regime at low gas velocity is usually not desired. The detection of this flow regime gives a clear indication that the diameters of the holes in the perforated plate should be decreased until a homogeneous regime is established at these low gas velocities.

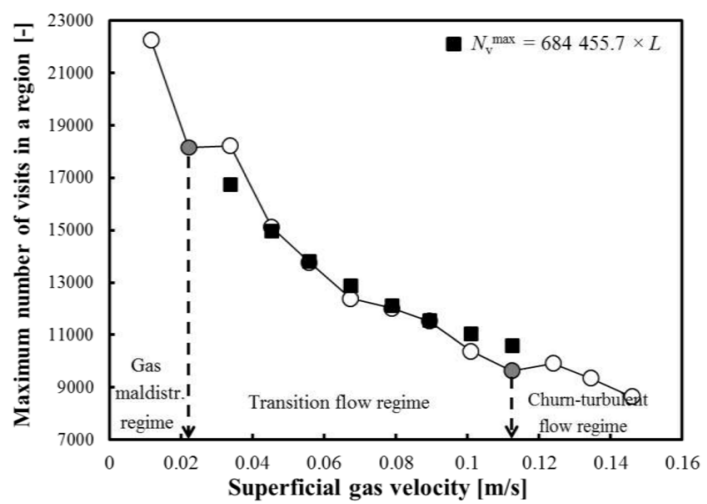

Fig. 4 Flow regime identification (based on the $N_{\mathrm{v}}^{\max }$ values) in a small bubble column $(0.15 \mathrm{~m}$ in i.d.) operated with an airwater system

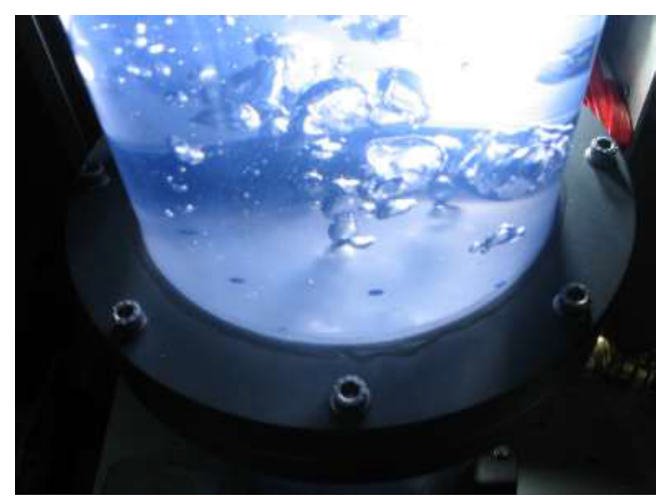

Fig. 5 Visual observation of gas maldistribution in a narrow bubble column $\left(0.15 \mathrm{~m}\right.$ in i.d.) operated at $U_{\mathrm{G}}=0.01 \mathrm{~m} / \mathrm{s}$

By using the total information entropy (extracted from differential pressure fluctuations), Nedeltchev and Shai$\mathrm{kh}$ (2013) identified two transition velocities in a bubble column $(0.102 \mathrm{~m}$ in i.d.) operated with nitrogen and tap water as gas and liquid phase, respectively. The column was equipped with a perforated plate distributor (19 holes, $\left.\varnothing 1 \times 10^{-3} \mathrm{~m}\right)$. At a height of $0.65 \mathrm{~m}$ above the gas distributor, the first transition velocity $U_{\text {trans }}$ was identified at $U_{\mathrm{G}}=0.025 \mathrm{~m} / \mathrm{s}$, while the second $U_{\text {trans }}$ was identified at $U_{\mathrm{G}}=0.037 \mathrm{~m} / \mathrm{s}$. Similar results were reported by Ajbar et al. (2009). They used a slightly larger bubble column $(0.15 \mathrm{~m}$ in i.d.) operated with an air-water system. The column was equipped with a ring sparger with six legs star-like cross $(85$ holes, $\varnothing 1 \times 10^{-3} \mathrm{~m}$ ). The authors reported that the first $U_{\text {trans }}$ occurred at $0.028 \mathrm{~m} / \mathrm{s}$, whereas the second transition took place at $0.047 \mathrm{~m} / \mathrm{s}$. The small differences could be attributed to the different gas spargers and column diameters. Except for the similar transition velocities, both papers report the existence of somewhat narrow transition regime.

In comparison with the previous results of Ajbar et al. (2009) and Nedeltchev and Shaikh (2013) it can be concluded that the first $U_{\text {trans }}$ value in Figure 4 is close to the first $U_{\text {trans }}$ values in their papers irrespective of the fact that the hole size $\left(\varnothing 4 \times 10^{-3} \mathrm{~m}\right)$ is much higher. It seems that the effect of the hole size is mostly pronounced on the 
second $U_{\text {trans }}$ value which is shifted to a much higher value $(0.112 \mathrm{~m} / \mathrm{s})$. In other words, the larger hole size stabilizes the transition flow regime and it expands it. In addition, at low $U_{\mathrm{G}}$ values a gas maldistribution flow regime is identified, which is normal for such a "coarse" gas distributor.

It is noteworthy that similar results (as compared to our study) were reported by Abbasi et al. (2013). In an air-water bubble column $(0.09 \mathrm{~m}$ in i.d.) equipped with a perforated plate distributor (196 holes, $\varnothing 0.5 \times 10^{-3} \mathrm{~m}, 0.6 \%$ open area) the authors identified two transition velocities at 0.025 and $0.1 \mathrm{~m} / \mathrm{s}$, respectively.

The monotonous decreasing trend in the transition flow regime can be used to determine the scale of the liquid mixing. It was found that the $N_{\mathrm{v}}{ }^{\max }$ values can be correlated to the mixing length $L$ as follows: $N_{\mathrm{v}}^{\max }=684,455.7 \times L$. In other words, the mixing length values calculated from Eq. (1) are very close to the $N_{\mathrm{v}}{ }^{\max }$ values in the transition flow regime when a proportionality constant is used (see Figure 4). The rate of decrease of both parameters (in this flow regime) as a function of superficial gas velocity $U_{\mathrm{G}}$ is quite similar. The average relative error between experimental and predicted $N_{\mathrm{v}}{ }^{\max }$ values (in the transition flow regime) is $3.87 \%$. This fact implies that the length scale of liquid mixing and the degree of liquid turbulence in the transition flow regime are proportional to the parameter introduced by Kawase and Tokunaga (1991). This correlation implies that in the above-defined transition flow regime the scale of liquid mixing varies from $2.45 \times 10^{-3} \mathrm{~m}$ down to $1.55 \times 10^{-3} \mathrm{~m}$, which is still much closer to the scale of the bubble diameter than to the scale of the column diameter.

In order to illustrate better how the signal values are distributed among the different regions, Figure 6 shows the distribution of number of visits by regions at $U_{\mathrm{G}}=0.022 \mathrm{~m} / \mathrm{s}$ (the first transition velocity). It can be seen that the range of the signal could be divided into 12 regions and that the middle region (No. 6) is the most frequently visited one. It has been visited 18,151 times (see the grey central box) and this value is used at $U_{\mathrm{G}}=0.022 \mathrm{~m} / \mathrm{s}$ in Figure 4 . The first region has a height of 0.25 (the gas holdup values are in percentage) and the height of every subsequent region increases stepwise with 0.25 . For instance, region 6 has a height of 1.5 and region 12 has a height of 3.0. Figure 6 shows that the first and the last regions are the least frequently visited ones. The number of visits in region 5 (with height 1.25) is comparable to the ones in region 6 . In a similar way, the values of $N_{\mathrm{v}}$ and $N_{\mathrm{v}}{ }^{\max }$ at the other superficial gas velocities have been determined.

The KE profile is shown in Figure 7. The same transition velocities $U_{\text {trans }}$ (as in Figure 4) are identified based on two local minima. Up to $U_{\mathrm{G}}=0.022 \mathrm{~m} / \mathrm{s}$ the $K E$ decreases and gas maldistribution was observed. Between $U_{\mathrm{G}}=0.022 \mathrm{~m} / \mathrm{s}$ and $U_{\mathrm{G}}=0.112 \mathrm{~m} / \mathrm{s}$ the transition flow regime is identified. In this hydrodynamic regime, the monotonous decrease of the $K E$ values could be correlated to the mixing length $L$ as follows: $K E=223.856 \times L$. The mixing length values $L$ are calculated by means of Eq. (1). The average relative error between experimental and predicted $K E$ values (in the

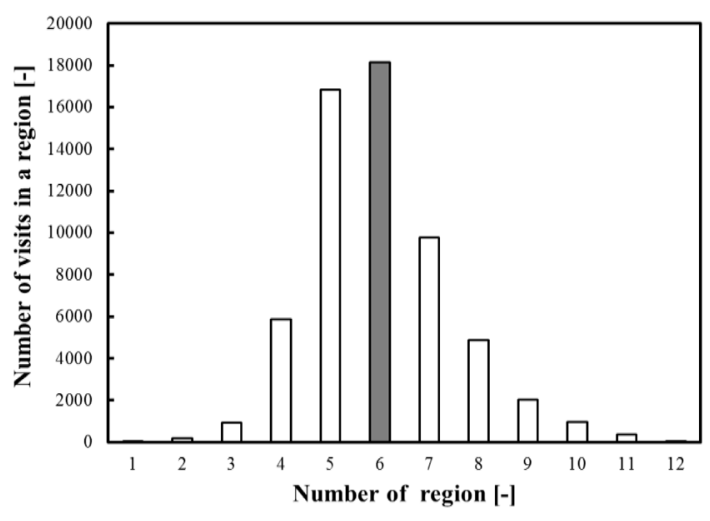

Fig. 6 Number of visits in different regions in a narrow bubble column $\left(0.15 \mathrm{~m}\right.$ in i.d.) operated at $U_{\mathrm{G}}=0.022 \mathrm{~m} / \mathrm{s}$

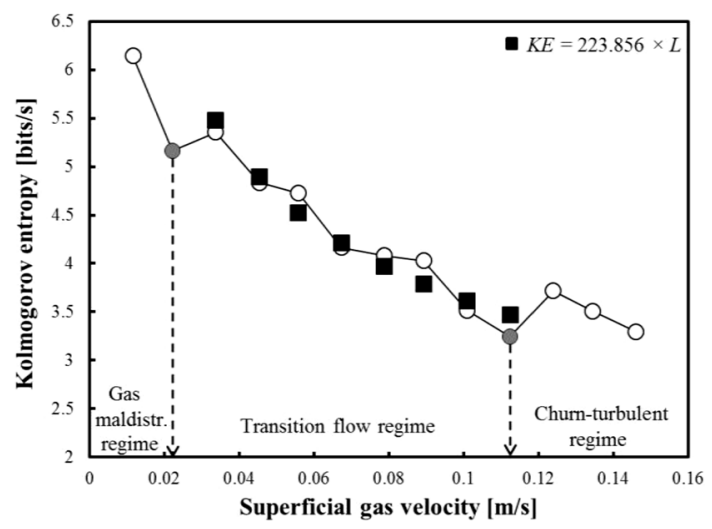

Fig. 7 Kolmogorov entropy $(K E)$ values in a small bubble column $(0.15 \mathrm{~m}$ in i.d.) operated with an air-water system

transition flow regime) is $3.42 \%$. Beyond $U_{\mathrm{G}}=0.112 \mathrm{~m} / \mathrm{s}$ the churn-turbulent flow regime is established. The explanation for the $K E$ minimum at each transition velocity is the same as for the $N_{\mathrm{v}}{ }^{\max }$ values, i.e. more structure is observed as suggested by Letzel et al. (1997).

It is worth noting that in each flow regime the $K E$ values are monotonously decreasing, which means that the gasliquid system is trying to reach a better ordered state that ultimately leads to the establishment of a new flow pattern (structure). There is a similarity in the profiles exhibited in Figures 4 and 7 although both parameters are completely independent.

In summary, a new parameter $\left(N_{\mathrm{v}}^{\max }\right)$ was introduced, which confirmed that the mixing scale in the transition regime is equivalent to the mixing length. This result was confirmed in terms of the $K E$ values. Both parameters lead to the conclusion that the mixing length concept is applicable only in the transition flow regime. This fact was not mentioned explicitly in the work of Kawase and Tokunaga (1991).

It is noteworthy that the two transition velocities $(0.022$ and $0.112 \mathrm{~m} / \mathrm{s}$ ) identified in this work might not be the most accurate values since they depend also on the selection of the unit height (in the case of $N_{\mathrm{v}}{ }^{\max }$ ) and both the cut-off length $L_{0}$ and the number of elements per vector as well as 
the delay time $\tau$ (in the case of $K E$ ).

Finally, it should be mentioned that the correlation of both parameters $\left(N_{\mathrm{v}}{ }^{\max }\right.$ and $\left.K E\right)$ to the mixing length could not be confirmed in a large bubble column ( $0.4 \mathrm{~m}$ in i.d.). Most likely this can be attributed to the enhanced turbulence in the large units which causes strong fluctuations in every signal.

In summary, by using the above-described algorithms for calculation of both $N_{\mathrm{v}}^{\max }$ and $K E$ (with the specified key parameters) the researchers can examine whether the concept of the mixing length is applicable in the transition flow regime in their facilities.

\section{Conclusion}

A new parameter called maximum number of visits per region $\left(N_{\mathrm{v}}{ }^{\max }\right)$ was defined and correlated to the mixing length in a narrow bubble column $(0.15 \mathrm{~m}$ in i.d.) operated with an air-water system in the transition flow regime. The liquid mixing in the transition regime was found to be at the scale of the mixing length which was independently confirmed by the KE profile. This limited applicability of the mixing length concept has not been reported in the literature hitherto.

Both parameters $\left(N_{\mathrm{v}}^{\max }\right.$ and $\left.K E\right)$ were capable of identifying two transition velocities $(0.022$ and $0.112 \mathrm{~m} / \mathrm{s})$. They delineated the boundaries of the three main flow regimes, i.e., gas maldistribution, transition and churn-turbulent. A unified criterion (local minimum) was applied for identification of the two main transition velocities.

\section{Acknowledgement}

Dr. Stoyan Nedeltchev gratefully acknowledges the financial support of the European Commission (Seventh Framework Program, Marie Curie Outgoing International Fellowship, Grant Agreement No. 221832). Dr. Markus Schubert gratefully acknowledges the financial support of the European Research Council (ERC Starting Grant, Grant Agreement No. 307360).

\section{Nomenclature}

$=$ number of sequential pair of points when the interpoint distance $<L_{0}$

$=$ bubble diameter

$D_{\mathrm{c}} \quad=$ column diameter

$E_{\mathrm{L}} \quad=$ liquid-phase axial dispersion coefficient

$f_{\mathrm{s}} \quad=$ sampling frequency

$K E \quad=$ Kolmogorov entropy

$L \quad=$ mixing length

$L_{0} \quad=$ maximum interpoint distance (cut-off length)

$M \quad=$ sample size of $b$ values

$m \quad=$ embedding dimension

$N \quad=$ number of data points

$N_{\mathrm{v}} \quad=$ number of visits in a region

$N_{\mathrm{v}}^{\max }=$ maximum number of visits per region

$U_{\mathrm{G}} \quad=$ superficial gas velocity

$U_{\text {trans }}=$ transitional gas velocity

$t_{0} \quad=$ time at the measurement start
$\Delta t \quad=$ sampling interval(s)

$\tau \quad=$ delay time

\section{Literature Cited}

Abbasi, M., N. Mostoufi, R. Sotudeh-Gharebagh and R. Zarghami; "A Novel Approach for Simultaneous Hydrodynamic Characterization of Gas-Liquid and Gas-Solid Systems," Chem. Eng. Sci., 100, 74-82 (2013)

Ajbar, A., W. Al-Masry and E. Ali; "Prediction of Flow Regime Transitions in Bubble Columns Using Passive Accoustic Measurements," Chem. Eng. Proc.: Proc. Intens., 48, 101-110 (2009)

Baird, M. H. I. and R. G. Rice; "Axial Dispersion in Large Unbaffled Columns," Chem. Eng. J., 9, 171-174 (1975)

Deckwer, W.-D., R. Burckhart and G. Zoll; "Mixing and Mass Transfer in Tall Bubble Columns," Chem. Eng. Sci., 29, 2177-2188 (1974)

Geary, N. W. and R. G. Rice; "Circulation in Bubble Columns: Corrections for Distorted Bubble Shape," AIChE J., 37, 1593-1594 (1991)

Geary, N. W. and R. G. Rice; "Circulation and Scale-Up in Bubble Columns," AIChE J., 38, 76-82 (1992)

Groen, J. S., R. G. C. Oldeman, R. F. Mudde and H. E. A. Van den Akker; "Coherent Structures and Axial Dispersion in Bubble Column Reactors," Chem. Eng. Sci., 51, 2511-2520 (1996)

Hinze, J. O.; Turbulence, McGraw-Hill, New York, U.S.A. (1975)

Kawase, Y. and M. Tokunaga; "Characteristic Mixing Length in Bubble Columns," Can. J. Chem. Eng., 69, 1228-1231 (1991)

Kawase, Y. and M. Moo-Young; "Liquid Phase Mixing in Bubble Columns with Newtonian and Non-Newtonian Fluids," Chem. Eng. Sci., 41, 1969-1977 (1986)

Kawase, Y. and M. Moo-Young; "Theoretical Prediction of Gas Holdup in Bubble Columns with Newtonian and Non-Newtonian Fluids," Ind. Eng. Chem. Res., 26, 933-937 (1987)

Letzel, H. M., J. C. Schouten, R. Krishna and C. M. van den Bleek; "Characterization of Regimes and Regime Transitions in Bubble Columns by Chaos Analysis of Pressure Signals," Chem. Eng. Sci., 52, 4447-4459 (1997)

Lübbert, A. and B. Larson; "Detailed Investigations of the Multiphase Flow in Airlift Tower Loop Reactors," Chem. Eng. Sci., 45, 30473053 (1990)

Nedeltchev, S.; "Application of Chaos Analysis for the Investigation of Turbulence in Heterogeneous Bubble Columns," Chem. Eng. Technol., 32, 1974-1983 (2009)

Nedeltchev, S. and A. Shaikh; "A New Method for Identification of the Main Transition Velocities in Multiphase Reactors Based on Information Entropy Theory," Chem. Eng. Sci., 100, 2-14 (2013)

Pandit, A. B. and J. B. Joshi; "Mass and Heat Transfer Characteristics of Three Phase Sparged Reactors," Chem. Eng. Res. Des., 64, 125-157 (1986)

Prasser, H.-M., A. Böttger and J. Zschau; "A New Electrode Tomograph for Gas-Liquid Flows," Flow Meas. Instrum., 9, 111-119 (1998)

Reilly, I. G., D. S. Scott, T. J. W. Debruijn and D. MacIntyre; “The Role of Gas Phase Momentum in Determining Gas Holdup and Hydrodynamic Flow Regimes in Bubble Column Operations," Can. J. Chem. Eng., 72, 3-12 (1994)

Rice, R. G. and M. A. Littlefield; "Dispersion Coefficients for Ideal Bubbly Flow in Truly Vertical Bubble Columns," Chem. Eng. Sci., 42, 2045-2053 (1987)

Rice, R. G. and N. W. Geary; "Prediction of Liquid Circulation in Viscous Bubble Columns," AIChE J., 36, 1339-1348 (1990)

Schlichting, H.; Boundary Layer Theory, McGraw-Hill, New York, 
U.S.A. (1968)

Schouten, J. C., F. Takens and C. M. van den Bleek; "Maximum-Likelihood Estimation of the Entropy of an Attractor," Phys. Rev. E Stat. Phys. Plasmas Fluids Relat. Interdiscip. Top., 49, 126-129 (1994a) Schouten, J. C., F. Takens and C. M. van den Bleek; "Estimation of the
Dimension of a Noisy Attractor," Phys. Rev. E Stat. Phys. Plasmas Fluids Relat. Interdiscip. Top., 50, 1851-1861 (1994b)

van den Bleek, C. M. and J. C. Schouten; "Deterministic Chaos: A New Tool in Fluidized Bed Design and Operation," Chem. Eng. J., 53, 75-87 (1993) 